\title{
Infrared photodetector based on GeTe nanofilms with high performance
}

\author{
Yiqun Zhao, ${ }^{1,4}$ Libin Tang, $, 1,2,3,{ }^{*}$ Shengy Yang, ${ }^{1, *}$ Kar Seng Teng,,${ }^{5, *}$ ShU \\ PING LAU 6 \\ ${ }^{1}$ School of Physics, Beijing Institute of Technology, Beijing 100081, China \\ ${ }^{2}$ Kunming Institute of Physics, Kunming 650223, China \\ 3 Yunnan Key Laboratory of Advanced Photoelectric Materials and Devices, Kunming 650223, China \\ ${ }^{4}$ Kunming Metallurgy College, Kunming 650033, China \\ ${ }^{5}$ College of Engineering, Swansea University, Bay Campus, Fabian Way, Swansea SA1 8EN, UK \\ ${ }^{6}$ Department of Applied Physics, The Hong Kong Polytechnic University, Hong Kong SAP, P.R. China \\ *Corresponding author: scitang@163.com; k.s.teng@swansea.ac.uk; syyang@bit.edu.cn
}

Received XX Month XXXX; revisedXX Month, XXXX; accepted XX MonthXXXX; posted XX Month XXXX (Doc. ID XXXXX); published XX Month XXXX

GeTe is an important narrow band gap semiconductor material, which has found application in the fields of thermoelectricity, phase change storage as well as switch. However, it has not been studied for application in the field of photodetector. Here, GeTe thin films were grown by magnetron sputtering and their material structure, optical and electrical properties were compared before and after annealing. High-performance photodetectors with detectivity of $\sim \mathbf{1 0}^{13}$ Jones at $850 \mathrm{~nm}$ light were demonstrated. Thus the novel application of GeTe in optoelectronic devices is reported in this work. (C) 2019 Optical Society of America

http://dx.doi.org/10.1364/OL.99.099999

GeTe and its compound materials have attracted extensive attention in recent years, in particular as efficient thermoelectric materials for midtemperature applications $[1,2]$. The absence of lead $(\mathrm{Pb})$ in such thermoelectric material presents itself as environment friendly material is highly favorable. The GeTe material has also been considered as a strong candidate for next-generation memory [3-11] and electrical switch technologies [12-15] due to its reversible phase change resulting in different electrical and optical properties. Furthermore, the potential application of GeTe in the field of computing has been explored recently [16]. The integration of the unique storage and computing features of GeTe will lead to groundbreaking technology that has major impact in the field of computing. As a narrow band gap semiconductor, GeTe material can potentially be used as infrared detectors. However, to the best knowledge of the authors, there is no report on its application in the field of photodetector. If a breakthrough is made in the application of GeTe in photodetector, it will become possible to facilitate the seamless connection between the two exciting fields of photoelectric detection and computing in the future. In this work, GeTe films were prepared by magnetron sputtering and the effect of post-annealing was investigated. The physical, electrical and optical properties of the films were studied.
A prototype GeTe photodetector was fabricated and evaluated for the first time.

The device was fabricated using the following processes. First, a quartz substrate was cleaned by chemical bath method with mixed solution containing $\mathrm{H}_{2} \mathrm{O}: \mathrm{H}_{2} \mathrm{O}_{2}: \mathrm{NH}_{3} \cdot \mathrm{H}_{2} \mathrm{O}(3: 1: 1)$ at $80^{\circ} \mathrm{C}$ for 30 min and dried under air flow. GeTe film was then deposited by magnetron sputtering directly onto the cleaned substrate at a power of $90 \mathrm{~W}$ and a pressure of $5 \mathrm{~Pa}$ for $120 \mathrm{~s}$ from an initial vacuum of $6.0 \times 10^{-4} \mathrm{~Pa}$ before sputtering. Subsequently, a pair of aluminum (Al) electrodes was evaporated onto the GeTe film using physical vapor deposition (PVD) technique (at a pressure of $7.0 \times 10^{-5} \mathrm{~Pa}$ ) through a shadow mask. The thickness of the $\mathrm{Al}$ electrodes was approximately $100 \mathrm{~nm}$ as monitored by a quartz oscillator during deposition. The effective area of the device was $3.0 \mathrm{~mm}^{2}$. Finally, the film was annealed in a vacuum oven at $260^{\circ} \mathrm{C}$ for $10 \mathrm{~min}$, which was based on previously reported phase transition temperature of the material in the literatures [11, 12, 15, 17].

Transmission electron microscopy (TEM) image of the as-deposited GeTe film is shown in Fig. 1 (a). According to the fast Fourier transform (FFT) pattern shown in Fig. 1 (b), the as-deposited GeTe film was amorphous [15, 18].

High-resolution TEM (HRTEM) image and electron diffraction pattern of the annealed GeTe film are shown in Fig. 1 (d) and (e), respectively. The indices of crystal planes of the film are indicated on the electron diffraction pattern. The results revealed crystalline GeTe film after annealing. Fig. 1 (f) shows the line profiles of the lattice fringes shown in Fig. 1 (d). The top and bottom line profiles of Fig. 1 (f) corresponded to (220) and (223) crystal planes of GeTe film, which exhibited a lattice fringe separation of 0.209 and $0.177 \mathrm{~nm}$, respectively. The angle between the two crystal planes was measured as $30.68^{\circ}$, which is in good agreement with a theoretical value of $30.51^{\circ}$. Hence, this suggests that the irradiation direction of the electron diffraction beam is of [1시 crystal orientation. The sketch of theoretical lattice of GeTe structure from the [1 $\overline{1} 0$ ] crystal orientation is illustrated in the inset of Fig. 1 (d). 

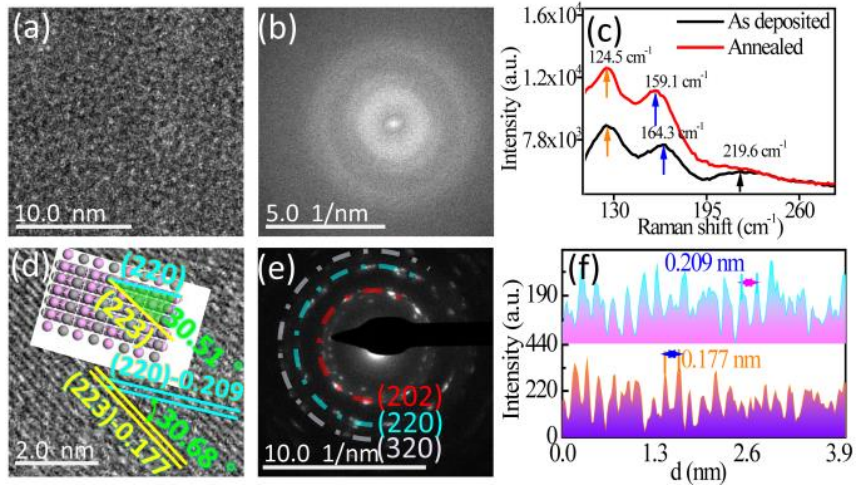

Fig. 1 (a) TEM image and (b) FFT pattern of the as-deposited GeTe film. (c) Raman spectra of the GeTe film before and after annealing. (d) TEM image of the annealed GeTe film and sketch of the crystal structure (inset). (e) Electron diffraction pattern of the annealed GeTe film. (f) Line profiles of the lattice fringes of (220) and (223) crystal planes as shown in the top and bottom, respectively.

Raman spectroscopy was performed to study the structure of the GeTe films before and after annealing using a Renishaw inVia Raman microscope with an argon-ion laser at an excitation wavelength of 514 nm. Fig. 1 (c) shows the Raman spectra of amorphous (black) and crystalline (red) GeTe films, which are in good agreement with the literatures $[19,20]$. There were three distinctive bands between the range of 100 and $280 \mathrm{~cm}^{-1}$. These bands were situated at $124.5,159$ and 219 $\mathrm{cm}^{-1}$. In the case of the amorphous film, as compared to the annealed film, the third band was more obvious, while the second band exhibited blueshift by $5.2 \mathrm{~cm}^{-1}$, and the first band was significantly smaller. After annealing, the third band has diminished, while the second band redshifted, and the first band has increased when compared to the asdeposited film due to structural transformation of GeTe film resulting in an expansion of crystallization range.

Atomic force microscopy (AFM) was carried out to determine the thickness of the films using AFM (SPA-400). Fig. 2 (a) and (b) reveal a thickness of $34 \pm 1.3$ and $35 \pm 1.4 \mathrm{~nm}$ for the as-deposited and annealed films on silicon dioxide substrates, respectively. Hence, the annealing process has little effect on the film thickness.

The effect of annealing on the structure of GeTe films was further investigated using X-ray diffraction (XRD). Fig. 2 (c) shows the XRD spectra of the as-deposited (blue) and annealed (red) GeTe films. It is clear that two diffraction peaks at $29.95^{\circ}$ and $43.51^{\circ}$, which corresponded to (202) and (220) lattice planes respectively, appeared after annealing the GeTe film. When combined with the above TEM results, it is evident that the GeTe film preferentially ordered along (220) lattice plane during the annealing process.

The elemental composition and chemical bonds of the GeTe films were studied by X-ray photoelectron spectroscopy (XPS) using AlKa radiation with energy of $1486.6 \mathrm{eV}$. XPS spectra of $\mathrm{Ge} 3 \mathrm{~d}$ and Te $3 \mathrm{~d}$ core level peaks of the as-deposited GeTe film are shown in Fig. 2 (d) and (e), respectively. The Ge $3 \mathrm{~d}$ peak was deconvoluted into two components, namely $\mathrm{Ge}$ and $\mathrm{GeO}_{2}$ at binding energy of 30.0 and 32.9 $\mathrm{eV}$, respectively. The Te $3 \mathrm{~d}$ core level consisted mainly of $\mathrm{Te} 3 \mathrm{~d}_{5 / 2}(572.4$ $\mathrm{eV})$ and $\mathrm{Te} 3 \mathrm{~d}_{3 / 2}(582.9 \mathrm{eV})$ doublet peaks. On the contrary to the asdeposited film, both Ge $3 \mathrm{~d}$ and Te $3 \mathrm{~d}$ core levels of annealed GeTe film exhibited oxygen-related components as shown in Fig. 2 (f) and (g), respectively. The $\mathrm{Te}^{4+}$ peaks at $576.5 \mathrm{eV}\left(\mathrm{Te} 3 \mathrm{~d}_{5 / 2}\right)$ and $587.0 \mathrm{eV}(\mathrm{Te}$ $3 \mathrm{~d}_{3 / 2}$ ) in Fig. $2(\mathrm{~g})$ were associated with $\mathrm{TeO}_{2}[21,22]$. While $\mathrm{GeO}_{2}$ and $\mathrm{TeO}_{2}$ were observed at the XPS spectra, they were absent from the XRD and TEM characterizations which suggested that the oxidation of $\mathrm{Ge}$ and
Te atoms were primarily localized at the surface of the film by atmospheric oxygen during the transfer and annealing process [21]. The results also suggested that the surface Ge atoms were more susceptible to oxidation in air than the surface Te atoms.
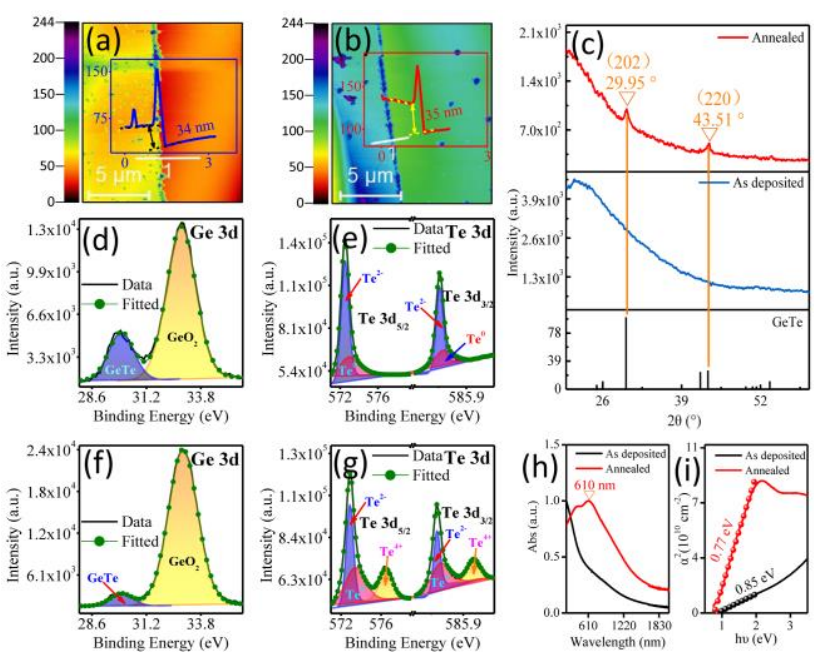

Fig. 2 (a)-(b) AFM images and line profiles (inset) for thickness measurement of the GeTe films before and after annealing, respectively. (c) XRD spectra of the GeTe films before and after annealing. (d)-(e) XPS spectra of Ge 3d and Te 3d core levels of as-deposited GeTe film. (f)-(g) XPS spectra of Ge $3 \mathrm{~d}$ and Te $3 \mathrm{~d}$ of annealed GeTe film. (h) Normalized UV-Vis-NIR absorption spectra of the GeTe films before and after annealing. (i) Plot of $\alpha^{2}$ versus photon energy $(h v)$ of the GeTe films before and after annealing.

To investigate the optical properties of the GeTe films before and after annealing, UV-Vis-NIR absorption spectroscopy were performed using a Horiba iHR 320 spectrometer. Fig. 2 (h) exhibits the normalized UVVis-NIR absorption spectra of both films. An absorption peak at $610 \mathrm{~nm}$ was apparent after annealing. The absorption coefficient of annealed GeTe film is significantly larger than that unannealed one. Furthermore in the infrared band, the longer the wavelength, the smaller the absorption coefficient. The band gap energy $\left(E_{g}\right)$ of the films can be determined using the following formulae [23, 24]:

$$
\alpha(h v)=\mathrm{C}\left(h v-E_{g}\right)^{1 / 2}
$$

where $h v$ is energy of incident photon, $\alpha$ is optical absorption coefficient associated with $h v$, and $\mathrm{C}$ is a constant. The $E_{g}$ of the GeTe films can be estimated from the curve of $\alpha^{2}$ vs. photon energy $(h v)$ as shown in Fig. 2 (i). The bandgap of GeTe can vary greatly depending on the experimental conditions and theoretical models [25]. In this work, the estimated $E_{g}$ for the GeTe films before and after annealing was $0.85 \mathrm{eV}$ and $0.77 \mathrm{eV}$, respectively. This is in good agreement with previous work performed by others, which reported an optical bandgap of $\sim 0.85 \mathrm{eV}$ for an amorphous GeTe film and $~ 0.73-0.95 \mathrm{eV}$ for crystalline film [26]. It showed a reduction of $E_{g}$ after annealing because of long-range ordering of the lattice. 
(a)
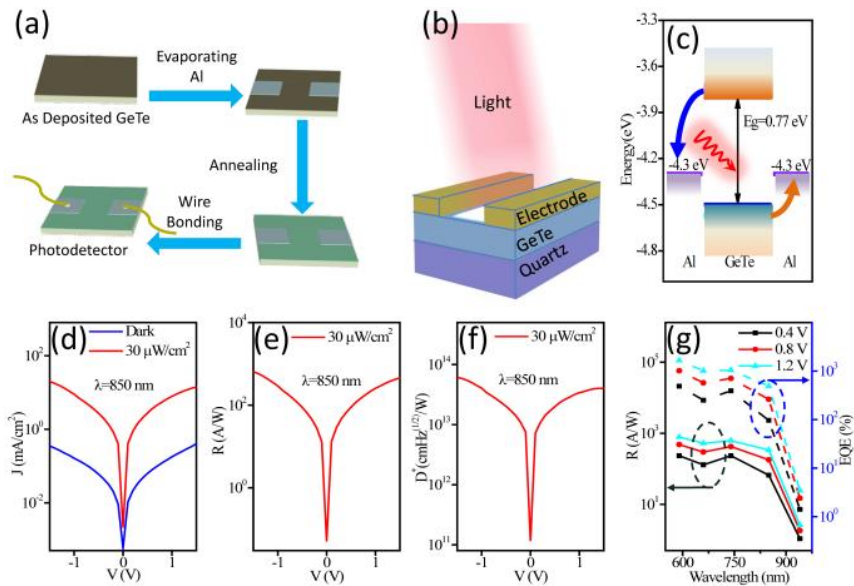

Fig. 3 (a) Schematic diagrams illustrating the fabrication process of photodetector based on GeTe film and (b) the device structure. (c) An energy band diagram illustrating the working principle of the photodetector. (d) Plot of $\log (J)-V$ of the photodetector under dark (blue line) and irradiation (red line) conditions. (e) Plot of $R$ (Responsivity)- $V$, (f) $D^{*}$ (Detectivity)- $V$ characteristic, and (g) Plots of $R$ (Responsivity) and EQE vs Wavelength of the GeTe based photodetector.

A prototype photodetector based on GeTe film was fabricated to explore the use of the material in the field of optoelectronics. GeTe film, which acted as the active layer of the photodetector, was deposited on to a quartz substrate by magnetron sputtering. A pair of $\mathrm{Al}$ electrodes was then evaporated onto the film using PVD technique. Finally, the device was annealed in a vacuum oven at $260{ }^{\circ} \mathrm{C}$ for $10 \mathrm{~min}$. The schematic diagrams illustrating the device fabrication process are shown in Fig. 3 (a). Fig. 3 (b) depicts the structure of the photodetector, which comprised of $\mathrm{GeTe}$ and $\mathrm{Al}$ with thicknesses of 35 and $100 \mathrm{~nm}$, respectively. The working principle of the photodetector is illustrated using an energy band diagram shown in Fig. 3 (c). The process can be divided into two steps as follows: (1) absorption of photons through the GeTe film, and (2) transfer of charge carriers to the $\mathrm{Al}$ electrodes.

Photoresponse of the photodetector was evaluated from $J-V$ measurements (using Keithley 2400 sourcemeter) under light illumination. The $\log (J)-V$ characteristics of the device irradiated by $\lambda=850 \mathrm{~nm}$ light at a density of $30 \mu \mathrm{W} \cdot \mathrm{cm}^{-2}$ and under dark condition performed at room temperature are shown in Fig. 3 (d). The results showed that the current density of the unoptimized device increased significant under irradiation that led to an exceptionally large ratio of photocurrent to dark current. This indicates a promising application of the GeTe film in infrared detection.

Responsivity $(R)$ and detectivity $\left(D^{*}\right)$ are two important figures of merit for photodetector. They can be determined by the equations below [27]:

$$
\begin{aligned}
R & =\frac{J_{p}}{P_{\text {opt }}} \\
D^{*} & =\frac{R}{\sqrt{2 \mathrm{q}\left|J_{d}\right|}}
\end{aligned}
$$

where $J_{p}$ is photocurrent that equals to absolute value of current density under irradiation subtracting that in the dark, $P_{\text {opt }}$ is incident optical power, $J_{d}$ is dark current density, and q is unit charge $\left(1.6 \times 10^{-19}\right.$ C).

The values of $R$ and $D^{*}$ can be obtained from Fig. 3 (e) and (f), respectively. The value of $R$ was between 88 and $650 \mathrm{~A} / \mathrm{W}$, and the value of $D^{*}$ was between $2 \times 10^{13}$ and $6 \times 10^{14}$ Jones $\left(1\right.$ Jones $=1 \mathrm{~cm} \cdot \mathrm{Hz}^{1 / 2} \mathrm{~W}^{-1}$ ) when the bias voltage was larger than $0.5 \mathrm{~V}$. There was slight fluctuation of $D^{*}$ as the device was not optimized and packaged. Fig. 3 (g) shows the plot of $R$ and external quantum efficiency (EQE) versus wavelength under different bias voltages. It can be seen that both $R$ and EQE are high, and they decrease with increasing wavelength in the infrared band. On the other hand, the unannealed device did not exhibit any photoresponse, which is mainly due to the amorphous nature of the unannealed GeTe film. Table 1 compares the responsivity and detectivity of infrared photodetectors based on different chalcogenide materials.

Table 1 Comparison of responsivity and detectivity of infrared photodetectors based on different chalcogenide materials

\begin{tabular}{cccc}
\hline Materials & $R(\mathrm{~A} / \mathrm{W})$ & $D^{*}$ (Jones) & Ref. \\
\hline $\mathrm{GeTe}$ & $\sim 10^{2}$ & $\sim 10^{13}$ & this work \\
$\mathrm{MoS}_{2}$ & $0.1 \sim 5.75 \times 10^{3}$ & & {$[28]$} \\
$\mathrm{MoS}_{2}$ & $9 \times 10^{2}$ & & {$[29]$} \\
$\mathrm{WSe}_{2}$ & $(1.8 \sim 3.5) \times 10^{5}$ & $>10^{14}$ & {$[30]$} \\
$\mathrm{In}_{2} \mathrm{Se}_{3}$ & $9.8 \times 10^{4}$ & $3.3 \times 10^{13}$ & {$[31]$} \\
$\mathrm{InSe}$ & $(1 \sim 5.68) \times 10^{4}$ & $1 \times 10^{13}$ & {$[32]$} \\
$\beta-\mathrm{Ga}_{2} \mathrm{O}_{3}$ & 1.68 & $3.73 \times 10^{10}$ & {$[33]$} \\
\hline
\end{tabular}

In conclusion, crystalline GeTe thin films were produced by magnetron sputtering and post-annealing. The physical, electronic and optical properties of the film before and after annealing were studied. After annealing GeTe film at $260^{\circ} \mathrm{C}$, the film revealed long-range order and band gap energy of $0.77 \mathrm{eV}$. Photodetector based on the annealed crystalline GeTe film was fabricated and showed high photoresponse at $850 \mathrm{~nm}$ irradiation with $R$ of $\sim 10^{2} \mathrm{~A} / \mathrm{W}$ and $D^{*}$ of $\sim 10^{13}$ Jones. In contrast, the photodetector with unannealed amorphous film did not show any photoresponse. The possibility of using GeTe in infrared detection was demonstrated in this work. It has enormous potential for integration with other fields, such as computing and storage, using GeTe based materials.

Funding. National Natural Science Foundation of China (Grant Nos. 61106098, and 51462037); Key Project of Applied Basic Research of Yunnan Province, China (GrantNo. 2012FA003); PolyU grants (1ZVGH and 1-BBAD); Research Grants Council of Hong Kong (Project Nos.: PolyU 153030/15P, PolyU 153271/16P, and PolyU 153039/17P)

Acknowledgment. We thank the Advanced Analysis and Measurement Center of Yunnan University for the use of their equipment.

Disclosures. The authors declare no conflicts of interest.

\section{References}

1. S. Perumal, S. Roychowdhury, and K. Biswas, J. Mater. Chem. C 4, 7520 (2016).

2. M. Hong, Z. G. Chen, L. Yang, Y. C. Zou, M. S. Dargusch, H. Wang, and J. Zou, Adv. Mater. 30, 1705942 (2018).

3. B. J. Kooi and J. Momand, physica status solidi (RRL) - Rapid Research Letters 13, 1800562 (2019).

4. M. Wuttig and N. Yamada, Nat. Mater. 6, 824 (2007).

5. E. Gourvest, B. Pelissier, C. Vallée, A. Roule, S. Lhostis, and S. Maitrejean, J. Electrochem. Soc. 159, H373 (2012).

6. W. Zhang, R. Mazzarello, M. Wuttig, and E. Ma, Nature Reviews Materials 4, 150 (2019).

7. S. Guerin, B. Hayden, D. W. Hewak, and C. Vian, ACS combinatorial science 19, 478 (2017). 
8. J. Tominaga, A. V. Kolobov, P. J. Fons, X. Wang, Y. Saito, T. Nakano, M. Hase, S. Murakami, J. Herfort, and Y. Takagaki, Science and technology of advanced materials 16, 014402 (2015).

9. A. V. Kolobov, M. Krbal, P. Fons, J. Tominaga, and T. Uruga, Nat. Chem. 3, 311 (2011).

10. D. Bang, H. Awano, J. Tominaga, A. V. Kolobov, P. Fons, Y. Saito, K. Makino, T. Nakano, M. Hase, Y. Takagaki, A. Giussani, R. Calarco, and S. Murakami, Sci. Rep. 4, 5727 (2014).

11. T. Siegrist, P. Jost, H. Volker, M. Woda, P. Merkelbach, C. Schlockermann, and M. Wuttig, Nat. Mater. 10, 202 (2011).

12. K. Ren, M. Zhu, W. Song, S. Lv, M. Xia, Y. Wang, Y. Lu, Z. Ji, and Z. Song, Nanoscale 11, 1595 (2019).

13. H. M. Aldosari, H. Simchi, Z. Ding, K. A. Cooley, S.-Y. Yu, and S. E. Mohney, ACS Appl. Mater. Interfaces 8, 34802 (2016).

14. N. El-Hinnawy, P. Borodulin, B. Wagner, M. R. King, J. S. Mason, E. B. Jones, S. McLaughlin, V. Veliadis, M. Snook, M. E. Sherwin, R. S. Howell, R. M. Young, and M. J. Lee, IEEE Electron Device Lett. 34, 1313 (2013).

15. M. R. King, B. P. Wagner, E. B. Jones, N. El-Hinnawy, P. Borodulin, S. R. McLaughlin, J. S. Mason, R. S. Howell, M. J. Lee, and R. M. Young, J. Vac. Sci. Technol. B 32, 041204 (2014).

16. C. Rinaldi, S. Varotto, M. Asa, J. Slawinska, J. Fujii, G. Vinai, S. Cecchi, D. Di Sante, R. Calarco, I. Vobornik, G. Panaccione, S. Picozzi, and R. Bertacco, Nano Lett. 18, 2751 (2018).

17. E. Carria, A. M. Mio, S. Gibilisco, M. Miritello, C. Bongiorno, M. G. Grimaldi, and E. Rimini, J. Electrochem. Soc. 159, H130 (2011).

18. X. Tian, T. Ma, L. Zhou, G. Brennecka, and X. Tan, J. Appl. Phys. 125, 245304 (2019).

19. K. S. Andrikopoulos, S. N. Yannopoulos, G. A. Voyiatzis, A. V. Kolobov, M. Ribes, and J. Tominaga, J. Phys.: Condens. Matter 18, 965 (2006).

20. D. Sarkar, G. Sanjeev, and M. G. Mahesha, Appl. Phys. A 119, 49 (2015).

21. L. V. Yashina, S. P. Kobeleva, T. B. Shatalova, V. P. Zlomanov, and V. I. Shtanov, Solid State lonics Diffusion \& Reactions 141, 513 (2001).

22. H. Qian, H. Tong, L. J. Zhou, B. H. Yan, H. K. Ji, K. H. Xue, X. M. Cheng, and X. S. Miao, J. Phys. D: Appl. Phys. 49, 495302 (2016).

23. J. S. Manser, J. A. Christians, and P. V. Kamat, Chem. Rev. 116, 12956 (2016).

24. J. Tauc and A. Menth, J. Non-Cryst. Solids 8-10, 569 (1972).

25. L. Vadkhiya, G. Arora, A. Rathor, and B. L. Ahuja, Radiat. Phys. Chem. 80, 1316 (2011).

26. S. K. Bahl and K. L. Chopra, J. Appl. Phys. 41, 2196 (1970).

27. P. Tian, L. Tang, J. Xiang, Z. Sun, R. Ji, S. K. Lai, S. Ping Lau, J. Kong, J. Zhao, C. Yang, and Y. Li, RSC Advances 6, 45166 (2016).

28. M. Long, P. Wang, H. Fang, and W. Hu, Adv. Funct. Mater. 29, 1803807 (2018).

29. D. Kufer, I. Nikitskiy, T. Lasanta, G. Navickaite, F. H. L. Koppens, and G. Konstantatos, Adv. Mater. 27, 176 (2015).

30. W. Zhang, M.-H. Chiu, C.-H. Chen, W. Chen, L.-J. Li, and A. T. S. Wee, ACS Nano 8, 8653 (2014).

31. J. O. Island, S. I. Blanter, M. Buscema, H. S. van der Zant, and A. Castellanos-Gomez, Nano Lett. 15, 7853 (2015).

32. W. Feng, J.-B. Wu, X. Li, W. Zheng, X. Zhou, K. Xiao, W. Cao, B. Yang, J.C. Idrobo, L. Basile, W. Tian, P. Tan, and P. Hu, J. Mater. Chem. C 3, 7022 (2015).

33. S. Oh, M. A. Mastro, M. J. Tadjer, and J. Kim, ECS Journal of Solid State Science and Technology 6, Q79 (2017). 


\section{The fifth page}

\section{Full references(to aid the editor and reviewers)}

1. S. Perumal, S. Roychowdhury, and K. Biswas, "High performance thermoelectric materials and devices based on GeTe," J. Mater. Chem. C 4, 7520 (2016).

2. M. Hong, Z. G. Chen, L. Yang, Y. C. Zou, M. S. Dargusch, H. Wang, and J. Zou, "Realizing zT of 2.3 in $\mathrm{Ge}_{1-x y} \mathrm{Sb}_{x} \operatorname{In}_{y}$ Te via Reducing the PhaseTransition Temperature and Introducing Resonant Energy Doping," Adv. Mater. 30, 1705942 (2018).

3. B. J. Kooi and J. Momand, "High Resolution Imaging of Chalcogenide Superlattices for Data Storage Applications: Progress and Prospects," physica status solidi (RRL) - Rapid Research Letters 13, 1800562 (2019).

4. M. Wuttig and N. Yamada, "Phase-change materials for rewriteable data storage," Nat. Mater. 6, 824 (2007).

5. E. Gourvest, B. Pelissier, C. Vallée, A. Roule, S. Lhostis, and S. Maitrejean, "Impact of Oxidation on $\mathrm{Ge}_{2} \mathrm{Sb}_{2} \mathrm{Te}_{5}$ and GeTe Phase-Change Properties," J. Electrochem. Soc. 159, H373 (2012).

6. W. Zhang, R. Mazzarello, M. Wuttig, and E. Ma, "Designing crystallization in phase-change materials for universal memory and neuro-inspired computing," Nature Reviews Materials 4, 150 (2019).

7. S. Guerin, B. Hayden, D. W. Hewak, and C. Vian, "Synthesis and Screening of Phase Change Chalcogenide Thin Film Materials for Data Storage," ACS combinatorial science 19, 478 (2017).

8. J. Tominaga, A. V. Kolobov, P. J. Fons, X. Wang, Y. Saito, T. Nakano, M. Hase, S. Murakami, J. Herfort, and Y. Takagaki, "Giant multiferroic effects in topological GeTe-Sb ${ }_{2} \mathrm{Te}_{3}$ superlattices," Science and technology of advanced materials 16, 014402 (2015).

9. A. V. Kolobov, M. Krbal, P. Fons, J. Tominaga, and T. Uruga, "Distortiontriggered loss of long-range order in solids with bonding energy hierarchy," Nat. Chem. 3, 311 (2011).

10. D. Bang, H. Awano, J. Tominaga, A. V. Kolobov, P. Fons, Y. Saito, K. Makino, T. Nakano, M. Hase, Y. Takagaki, A. Giussani, R. Calarco, and S. Murakami, "Mirror-symmetric magneto-optical Kerr rotation using visible light in $\left[(\mathrm{GeTe})_{2}\left(\mathrm{Sb}_{2} \mathrm{Te}_{3}\right)_{1}\right]_{n}$ topological superlattices," Sci. Rep. 4, 5727 (2014).

11. T. Siegrist, P. Jost, H. Volker, M. Woda, P. Merkelbach, C. Schlockermann, and M. Wuttig, "Disorder-induced localization in crystalline phase-change materials," Nat. Mater. 10, 202 (2011).

12. K. Ren, M. Zhu, W. Song, S. Lv, M. Xia, Y. Wang, Y. Lu, Z. Ji, and Z. Song, "Electrical switching properties and structural characteristics of GeSeGeTe films," Nanoscale 11, 1595 (2019).

13. H. M. Aldosari, H. Simchi, Z. Ding, K. A. Cooley, S.-Y. Yu, and S. E. Mohney, "Impact of Premetallization Surface Preparation on Nickelbased Ohmic Contacts to Germanium Telluride: An X-ray Photoelectron Spectroscopic Study," ACS Appl. Mater. Interfaces 8, 34802 (2016).

14. N. El-Hinnawy, P. Borodulin, B. Wagner, M. R. King, J. S. Mason, E. B. Jones, S. McLaughlin, V. Veliadis, M. Snook, M. E. Sherwin, R. S. Howell, R. M. Young, and M. J. Lee, "A Four-Terminal, Inline, Chalcogenide Phase-Change RF Switch Using an Independent Resistive Heater for Thermal Actuation," IEEE Electron Device Lett. 34, 1313 (2013).

15. M. R. King, B. P. Wagner, E. B. Jones, N. El-Hinnawy, P. Borodulin, S. R. McLaughlin, J. S. Mason, R. S. Howell, M. J. Lee, and R. M. Young, "Development of cap-free sputtered GeTe films for inline phase change switch based RF circuits," J. Vac. Sci. Technol. B 32, 041204 (2014).

16. C. Rinaldi, S. Varotto, M. Asa, J. Slawinska, J. Fujii, G. Vinai, S. Cecchi, D. Di Sante, R. Calarco, I. Vobornik, G. Panaccione, S. Picozzi, and R. Bertacco, "Ferroelectric Control of the Spin Texture in GeTe," Nano Lett. 18, 2751 (2018).

17. E. Carria, A. M. Mio, S. Gibilisco, M. Miritello, C. Bongiorno, M. G. Grimaldi, and E. Rimini, "Amorphous-Crystal Phase Transitions in $\mathrm{Ge}_{x} \mathrm{Te}_{1-\mathrm{x}}$ Alloys," J. Electrochem. Soc. 159, H130 (2011).
18. X. Tian, T. Ma, L. Zhou, G. Brennecka, and X. Tan, "In situ TEM study of the transitions between crystalline $\mathrm{Si}$ and nonstoichiometric amorphous oxide under bipolar voltage bias," J. Appl. Phys. 125, 245304 (2019).

19. K. S. Andrikopoulos, S. N. Yannopoulos, G. A. Voyiatzis, A. V. Kolobov, M. Ribes, and J. Tominaga, "Raman scattering study of the a-GeTe structure and possible mechanism for the amorphous to crystal transition," J. Phys.: Condens. Matter 18, 965 (2006).

20. D. Sarkar, G. Sanjeev, and M. G. Mahesha, "Analysis of electron beaminduced effect on electrical switching properties of glass chalcogenide GeTe thin films through Raman spectroscopy," Appl. Phys. A 119, 49 (2015).

21. L. V. Yashina, S. P. Kobeleva, T. B. Shatalova, V. P. Zlomanov, and V. I. Shtanov, "XPS study of fresh and oxidized GeTe and (Ge,Sn)Te surface," Solid State lonics Diffusion \& Reactions 141, 513 (2001).

22. H. Qian, H. Tong, L. J. Zhou, B. H. Yan, H. K. Ji, K. H. Xue, X. M. Cheng, and X. S. Miao, "Low work function of crystalline $\mathrm{GeTe} / \mathrm{Sb}_{2} \mathrm{Te}_{3}$ superlattice-like films induced by Te dangling bonds," J. Phys. D: Appl. Phys. 49, 495302 (2016).

23. J. S. Manser, J. A. Christians, and P. V. Kamat, "Intriguing Optoelectronic Properties of Metal Halide Perovskites," Chem. Rev. 116, 12956 (2016).

24. J. Tauc and A. Menth, "States in the gap," J. Non-Cryst. Solids 8-10, 569 (1972).

25. L. Vadkhiya, G. Arora, A. Rathor, and B. L. Ahuja, " Electron momentum

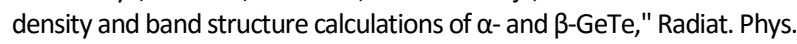
Chem. 80, 1316 (2011).

26. S. K. Bahl and K. L. Chopra, "Amorphous versus Crystalline GeTe Films. III. Electrical Properties and Band Structure," J. Appl. Phys. 41, 2196 (1970).

27. P. Tian, L. Tang, J. Xiang, Z. Sun, R. Ji, S. K. Lai, S. Ping Lau, J. Kong, J. Zhao, C. Yang, and Y. Li, "Solution processable high-performance infrared organic photodetector by iodine doping," RSC Advances 6 , 45166 (2016).

28. M. Long, P. Wang, H. Fang, and W. Hu, "Progress, Challenges, and Opportunities for 2D Material Based Photodetectors," Adv. Funct. Mater. 29, 1803807 (2018).

29. D. Kufer, I. Nikitskiy, T. Lasanta, G. Navickaite, F. H. L. Koppens, and G. Konstantatos, "Hybrid 2D-OD MoS 2 -PbS Quantum Dot Photodetectors," Adv. Mater. 27, 176 (2015).

30. W. Zhang, M.-H. Chiu, C.-H. Chen, W. Chen, L.-J. Li, and A. T. S. Wee, "Role of Metal Contacts in High-Performance Phototransistors Based on WSe $e_{2}$ Monolayers," ACS Nano 8, 8653 (2014).

31. J. O. Island, S. I. Blanter, M. Buscema, H. S. van der Zant, and A. Castellanos-Gomez, "Gate Controlled Photocurrent Generation Mechanisms in High-Gain $\ln _{2} \mathrm{Se}_{3}$ Phototransistors," Nano Lett. 15, 7853 (2015).

32. W. Feng, J.-B. Wu, X. Li, W. Zheng, X. Zhou, K. Xiao, W. Cao, B. Yang, J.C. Idrobo, L. Basile, W. Tian, P. Tan, and P. Hu, "Ultrahigh photoresponsivity and detectivity in multilayer InSe nanosheets phototransistors with broadband response," J. Mater. Chem. C 3, 7022 (2015).

33. S. Oh, M. A. Mastro, M. J. Tadjer, and J. Kim, "Solar-Blind MetalSemiconductor-Metal Photodetectors Based on an Exfoliated $\beta-\mathrm{Ga}_{2} \mathrm{O}_{3}$ Micro-Flake," ECS Journal of Solid State Science and Technology 6, Q79 (2017). 